\title{
ON THE NUMBER OF SQUARES IN A GROUP
}

\author{
LUDOMIR NEWELSKI
}

\begin{abstract}
We show that there is a connection between the number of squares in a group and the cardinality of the group. For example, if a group has countably many squares and $x^{2}=e$ implies $x=e$, then its cardinality is bounded by $2^{\aleph_{0}}$ and this bound can be obtained.
\end{abstract}

0. Introduction. This paper gives a negative answer to the following conjecture: If in a group without elements of order 2 there are only countably squares, then this group is countable.

In all the paper we deal with infinite groups only. In $\S 1$ we prove that if $\kappa$ is an infinite cardinal and a group $G$ without elements of order 2 has $\kappa$ many squares, then the cardinality of $G$ is bounded by $2^{\kappa}$. The proof is technical and uses the Erdős-Rado theorem. In $\S 2$ we construct an example of a torsion-free group $G$ of power continuum with only countably many squares, which according to the results from $\S 1$ is the best possible. The construction easily generalizes (Corollary 2.5) to the case of $\lambda$-many squares where $\lambda$ is an arbitrary infinite cardinal; however in this case we are able to construct a group whose cardinality is that of a linear ordering with a dense subset of cardinality $\lambda$.

One could ask if the above results easily generalize when squares are replaced by higher powers. For $k>1$ let $G^{k}=\left\{x^{k}: x \in G\right\}$. When dealing with $k$ th powers, the natural assumption on a group is that $x^{k}=e$ implies $x=e$. After the first version of this paper was written, Professor G. Higman pointed out to the author the following:

(1) If we want to prove for some $f$ satisfying $f(\kappa) \geq 2^{\kappa}$ that, for any $G,|G| \leq$ $f\left(\left|G^{k}\right|\right)$, then w.l.o.g. we may assume that $G^{k}$ is contained in the center of $G$.

(2) If Burnside's group of exponent $k$ with 2 generators is finite, then for any $G$ satisfying $x^{k}=e \rightarrow x=e$ we have $|G| \leq 2^{\left|G^{k}\right|}$.

Thus (2) provides us with another, maybe more algebraic, proof of Theorem 1.3, and gives us an estimation for $G$ in case $k=2,3,4,6$. However, (1) enabled the author to strengthen his previous estimations and prove that $|G| \leq 2^{\mid G^{k}}$ for $k=2^{n} 3^{m}$ where $n \geq 0$ and $m=0$ or 1 . For other $k$ 's the question of whether any estimation exists remains at present open. However, at least we cannot prove that for some $k>1$, for any $G,|G|=\left|G^{k}\right|$, as the construction from $\S 2$ generalizes to the case of $k$ th powers for any $k>2$. In contrast to the situation with infinite groups, we trivially have that if $G^{k}$ is finite and $G$ satisfies $x^{k}=e \rightarrow x=e$, then $G=G^{k}$. Notice that constructing for some $k$ a group $G$ such that $|G|>2^{\left|G^{k}\right|}$ (and $x^{k}=e \rightarrow x=e$ holds) would enable us to prove that Burnside's group of exponent $k$ is infinite.

Received by the editors January 31, 1985 and, in revised form, May 1, 1985 and November 1, 1985.

1980 Mathematics Subject Classification (1985 Revision). Primary 20E34; Secondary 20 F05. 
We use the standard set-theoretical notation. So, for example, ${ }^{<\omega} X$ denotes the set of all finite sequences of elements of $X$. For a finite sequence $\vec{\eta}, \iota(\vec{\eta})$ denotes the length of $\vec{\eta}$.

1. Negative results. Throughout this section we assume that $G$ is a group without elements of order 2.

FACT 1.1 (A SPECIAL CASE OF THE ERDŐS-RADO THEOREM, SEE [1, 3]). Let $\mu$ be an infinite cardinal. Assume that $f$ is a binary function on $\left(2^{\mu}\right)^{+}$ such that for every $\alpha<\beta<\left(2^{\mu}\right)^{+}$we have $f(\alpha, \beta) \in \mu$. Then there exists $\delta<\mu$ and $A \subseteq\left(2^{\mu}\right)^{+},|A|=\mu^{+}$, such that, for every $\alpha, \beta \in A$, if $\alpha<\beta$, then $f(\alpha, \beta)=\delta$.

LEMMA 1.2. Assume that $a, b, c, d \in G,(a b)^{2}=(c d)^{2},(b c)^{2}=(b d)^{2}$, and $a^{2}=b^{2}=c^{2}=d^{2}$. Then $a=b$ and $c=d$.

ProOF. We have $b c b c=b d b d$, so $c b c=d b d$. Multiplying this equation on the right side by $d$ and on the left by $c$ we obtain $c^{2} b c d=c d b d^{2}$. From the assumptions it follows that $c^{2}$ commutes with $b, c, d$, and equals $d^{2}$, so we get $b c d=c d b$. Now $(a b)^{2} b=(c d)^{2} b=b(c d)^{2}=b(a b)^{2}$. So $(a b)^{2} b=b(a b)^{2}=b a b a b=(b a)^{2} b$, hence $(a b)^{2}=(b a)^{2}$. From this we get

$$
(a b)^{4}=(a b)^{2}(b a)^{2}=a b a b b a b a=b^{2} b^{2} a^{2} a^{2}=a^{8} .
$$

Hence $\left(a^{-2} a b\right)^{4}=e$. But in $G$ there are no elements of order 2 , so $a^{-2} a b=a^{-1} b=$ $e$. We conclude that $a=b$. Repeating the above proof for $c, d, a, b$ instead of $a, b, c, d$, we see that $c=d$.

THEOREM 1.3. Assume that $G$ is an infinite group. Then $|G| \leq 2^{\left|G^{2}\right|}$.

PROOF. By the remark in the Introdution we can assume that $G^{2}$ is infinite. Let $\left|G^{2}\right|=\mu$. Suppose that $|G|>2^{\mu}$. Then we can find in $G$ pairwise distinct elements $\left\{a_{\alpha}: \alpha<\left(2^{\mu}\right)^{+}\right\}$such that $a_{\alpha}^{2}=a_{\beta}^{2}$ for every $\alpha<\beta<\left(2^{\mu}\right)^{+}$. For $\alpha<\beta<\left(2^{\mu}\right)^{+}$let $f(\alpha, \beta)=\left(a_{\alpha} a_{\beta}\right)^{2} \in G^{2}$. From Fact 1.1 it follows that we can choose an infinite set $A \subseteq\left(2^{\mu}\right)^{+}$such that for $\alpha, \beta, \alpha^{\prime}, \beta^{\prime} \in A$ if $\alpha<\beta, \alpha^{\prime}<\beta^{\prime}$ then $f(\alpha, \beta)=f\left(\alpha^{\prime}, \beta^{\prime}\right)$. W.l.o.g. we can assume that $\left\{a_{n}: n<\omega\right\} \subseteq A$. So we have $a_{0}^{2}=a_{1}^{2}=a_{2}^{2}=a_{3}^{2},\left(a_{0} a_{1}\right)^{2}=\left(a_{2} a_{3}\right)^{2}$, and $\left(a_{1} a_{2}\right)^{2}=\left(a_{1} a_{3}\right)^{2}$. From Lemma 1.2 we conclude that $a_{0}=a_{1}$ and $a_{2}=a_{3}$, a contradiction.

DEFINITION 1.4. For a given complete theory $T$ in a first-order language $L$, a formula $\varphi(\bar{x} ; \bar{y})$ of $L$ is stable in $T$ if for every model $M$ of $T$ there are $\leq\|M\|$ $\varphi$-types over $M$. If $\varphi$ is not stable, we call it unstable.

For basic results on stability, see, for example, [2, or 3]. If $G$ is a group, then we say that $\varphi(\bar{x} ; \bar{y})$ is stable in $G$ instead of "stable in $T=\operatorname{Th}(G)$."

THEOREM 1.5. Assume that $G$ is an infinite group. If $\varphi(x ; y) \equiv x y=y x$ is stable in $G$, then $|G|=\left|G^{2}\right|$.

Proof. If not, let $\left|G^{2}\right|=\mu$, and choose $\left\{a_{\alpha}: \alpha<\mu^{+}\right\} \subseteq G$ such that $a_{\alpha}^{2}=a_{\beta}^{2}$ for $\alpha<\beta<\mu^{+}\left(\mu \geq \aleph_{0}\right.$ by Theorem 1.3).

By induction one can easily construct three sequences $\left\{b_{n}\right\}_{n<\omega},\left\{c_{n}\right\}_{n<\omega} \subseteq G$ and $\left\{B_{n}\right\}_{n<\omega}, B_{n} \subseteq G$ for every $n$, such that

(1) $b_{0}=a_{0}$

(2) $b_{n} \in B_{n} \backslash B_{n+1}, B_{n+1} \subseteq B_{n} \subseteq\left\{a_{\alpha}: \alpha<\mu^{+}\right\}$for $n<\omega$,

(3) $\left|B_{n}\right|=\mu^{+}$, 
(4) if $c, d \in B_{n+1}$ then $\left(b_{n} c\right)^{2}=\left(b_{n} d\right)^{2}=c_{n} \in G^{2}$.

Now if there are $n<m$ such that $c_{2 n}=c_{2 m}$, then taking $b_{2 n}, b_{2 n+1}, b_{2 m}, b_{2 m+1}$ we see that

$$
\left(b_{2 n} b_{2 n+1}\right)^{2}=\left(b_{2 m} b_{2 m+1}\right)^{2}, \quad\left(b_{2 n+1} b_{2 m+1}\right)^{2}=\left(b_{2 n+1} b_{2 m}\right)^{2}
$$

and

$$
b_{2 n}^{2}=b_{2 n+1}^{2}=b_{2 m}^{2}=b_{2 m+1}^{2} .
$$

So Lemma 1.2 leads to a contradiction. Thus for every $n<m, c_{2 n} \neq c_{2 m}$. From this we get that, for $n \neq m,\left(b_{4 n} b_{4 m}\right)^{2}=\left(b_{4 n} b_{4 m+2}\right)^{2}$ holds if and only if $n<m$. But $\left(b_{4 n} b_{4 m}\right)^{2}=\left(b_{4 n} b_{4 m+2}\right)^{2}$ is equivalent to $b_{4 n} b_{4 m} b_{4 m+2}=b_{4 m} b_{4 m+2} b_{4 n}$, so to $\varphi\left(b_{4 n} ; b_{4 m} b_{4 m+2}\right)$. It follows that using $\varphi(x ; y)$ we can define on some infinite subset of $G$ a linear order. From the facts proved in [3] we can easily deduce that $\varphi(x ; y)$ is unstable in $G$.

2. An example. In this section we shall construct an example of a group $G$ of power continuum, without elements of order 2, with only countably many squares. From Theorem 1.3 it follows that such an example is the best possible for a countable $G^{2}$, i.e. $|G|$ is maximal possible.

The construction consists of defining a suitable normal subgroup $H_{0}$ of a free group $G_{0}$ of power continuum. $G$ will be the quotient group $G_{0} / H_{0}$. Let $G_{0}$ be the free group generated by the set of letters $\left\{\eta: \eta \in{ }^{\omega} 2\right\}$. We identify finite sequences of elements of ${ }^{\omega} 2$ (i.e. elements of $<\omega\left({ }^{\omega} 2\right)$ ) with appropriate words - element of $G_{0}$. Recall that $k=\{i: i<k\}$ for $k \in \omega$.

Definition 2.1. For every $\vec{\eta} \in<{ }^{<\omega}\left({ }^{\omega} 2\right)$ we define $\vec{h}(\vec{\eta}) \in<\omega(<\omega 2)$ as follows: Let $\iota(\vec{\eta})=n$. For $i<n$ let

$$
k_{i}=\max _{i \neq j<n} \min \{t+1: t<\omega \& \vec{\eta}[i]|t \neq \vec{\eta}[j]| t\} .
$$

Here $\min \varnothing=\max \varnothing=0$. Define $\vec{h}(\vec{\eta})[i]=\vec{\eta}[i] \uparrow k_{i}$ for every $i<n$, and $\iota(\vec{h}(\vec{\eta}))=n$.

We define now a subgroup $H_{0}$ of $G_{0}$ as follows:

DEFINITION 2.2. (1) $R=\left\{(\vec{\eta})^{2}(\vec{\nu})^{-2}: \vec{\eta}, \vec{\nu} \in<\omega\left(\omega_{2}\right)\right.$ \& $\vec{h}(\vec{\eta})=$ $\vec{h}(\vec{\nu})\}$.

(2) Let $H_{0}$ be the normal subgroup of $G_{0}$ generated by $R$, and let $G=G_{0} / H_{0}$.

For notational simplicity we treat words in $G_{0}$ as names for their images in $G$ under the canonical projection. 2.

THEOREM 2.3. $|G|=2^{\aleph_{0}},\left|G^{2}\right|=\aleph_{0}$ and in $G$ there are no elements of order

We prove Theorem 2.3 in a series of lemmas.

LemMa 1. If $\eta, \nu \in{ }^{\omega} 2, \eta \neq \nu$ then, in $G, \eta \neq \nu$.

Proof. Consider a homomorphism $f: G_{0} \rightarrow \mathbf{Z}_{2}$ such that $f(\eta)=0$ and $f(\nu)=$ 1. Then $H_{0} \subseteq \operatorname{Ker} f .0 \neq 1$, so, in $G=G_{0} / H_{0}, \eta \neq \nu$.

Notice that if $\eta, \nu \in{ }^{\omega} 2, \eta \neq \nu$, then $\vec{h}(\langle\eta\rangle)=\vec{h}(\langle\nu\rangle)=\langle\varnothing\rangle$, so in $G$ we have $\eta^{2}=\nu^{2}$. Denote this common square by $\alpha$, i.e. for all $\eta \in{ }^{\omega} 2, \eta^{2}=\alpha$ in $G$. 
LEMMA 2. Every element of $G$ is equal to $\alpha^{k} \vec{\eta}$ for some $k \in \mathbf{Z}$ and $\vec{\eta} \in$ $<\omega\left({ }^{\omega} 2\right)$ such that, for $0 \leq i<\iota(\vec{\eta})-1, \vec{\eta}[i] \neq \vec{\eta}[i+1]$.

Proof. Notice that for $\eta \in{ }^{\omega} 2$, in $G, \eta^{-1}=\alpha^{-1} \eta ; \eta \eta=\alpha$; and $\alpha, \alpha^{-1}$ commute with $\nu, \nu^{-1}$ for every $\nu \in{ }^{\omega} 2$.

LEMMA 3. $\left|G^{2}\right| \leq \aleph_{0}$.

ProOF. The value of $\left(\alpha^{k} \vec{\eta}\right)^{2}=\alpha^{2 k}(\vec{\eta})^{2}$ depends only on $k$ and $\vec{h}(\vec{\eta})$, so there are only countably many possibilities.

LEMMA 4. In $G$ : If $\left(\alpha^{k} \vec{\eta}\right)^{2}=e$, then $\alpha^{k} \vec{\eta}=e$.

Proof. We prove it by induction on $|\operatorname{Rng}(\vec{\eta})|$. We may assume that $\vec{\eta}$ is such as in Lemma 2.

(a) If $|\operatorname{Rng}(\vec{\eta})|=0$ then $\vec{\eta}=\varnothing$, and we have $\left(\alpha^{k}\right)^{2}=\alpha^{2 k}=e$.

(b) If $|\operatorname{Rng}(\vec{\eta})|=1$ then $\vec{\eta}=\langle\eta\rangle$ for some $\eta \in{ }^{\omega} 2$, so we have

$$
\left(\alpha^{k} \vec{\eta}\right)^{2}=\alpha^{2 k} \eta \eta=\alpha^{2 k+1} .
$$

Consider now the homomorphism $f: G_{0} \rightarrow(\mathbf{Z},+)$, such that, for every $\eta \in{ }^{\omega} \mathbf{2}$, $f(\eta)=1$ in $\mathbf{Z}$. Then clearly $H_{0} \subseteq \operatorname{Ker} f$, so whenever $f(x) \neq f(y)$ in $\mathbf{Z}, x \neq y$ holds in $G$. (For simplicity we do not distinguish explicitly between $f$ and the induced homomorphism $\bar{f}: G \rightarrow(\mathrm{Z},+)$.) So in case (a) we get $f\left(\alpha^{2 k}\right)=4 k=0$, hence $k=0$. Similarly, in case (b) we get $4 k+2=0$, a contradiction.

(c) Assume that $|\operatorname{Rng}(\vec{\eta})|=n \geq 2$, and for all $\alpha^{\imath} \vec{\nu}$ such that $|\operatorname{Rng}(\vec{\nu})|<n$, Lemma 4 holds. Assume that $\left.\alpha^{k} \vec{\eta}\right)^{2}=e$. We have to prove that $\alpha^{k} \vec{\eta}=e$.

Let us choose two letters $\eta, \nu \in \operatorname{Rng}(\vec{\eta})$ such that

$$
\iota(\vec{h}(\langle\eta, \nu\rangle)[0])=\iota(\vec{h}(\langle\eta, \nu\rangle)[1])
$$

is maximal possible. If there are two or more such pairs $\eta, \nu \in \operatorname{Rng}(\vec{\eta})$, we choose one of them. We prove that there is an inner automorphism of $G$ which maps $\alpha^{k} \vec{\eta}$ to some $\alpha^{\iota} \vec{\eta}_{1}$ such that $\left(\alpha^{\iota} \vec{\eta}_{1}\right)^{2}=e$ and $\left|\operatorname{Rng}\left(\vec{\eta}_{1}\right)\right|<n$. Clearly by the induction hypothesis this will finish the proof of Lemma 4.

Claim A. If $\vec{\eta}=\vec{\eta}_{0} \frown\langle\eta\rangle-\vec{\eta}_{1} \frown\langle\eta\rangle-\vec{\eta}_{2}$, and $\eta, \nu \notin \operatorname{Rng}\left(\vec{\eta}_{1}\right)$, then, in $G$, $\eta \vec{\eta}_{1} \eta=\nu \vec{\eta}_{1} \nu$

PROOF OF Claim A. Notice that from the maximality of the choice of $\eta, \nu$ and from the definition of $\vec{h}$ it follows that

$$
\vec{h}\left(\vec{\eta}_{1} \frown\langle\eta\rangle\right)=\vec{h}\left(\vec{\eta}_{1} \frown\langle\nu\rangle\right) \text {. }
$$

So in $G, \vec{\eta}_{1} \eta \vec{\eta}_{1} \eta=\vec{\eta}_{1} \nu \vec{\eta}_{1} \nu$. Multiplying on the left side by $\left(\vec{\eta}_{1}\right)^{-1}$ we see that Claim A holds.

From Claim A follows at once

Claim B. If $\vec{\eta}=\vec{\eta}_{0}-\langle\eta\rangle-\vec{\eta}_{1}-\langle\nu\rangle-\vec{\eta}_{2}-\langle\nu\rangle-\vec{\eta}_{3}$ and $\eta, \nu \notin \operatorname{Rng}\left(\vec{\eta}_{1}-\vec{\eta}_{2}\right)$ then, in $G, \vec{\eta}=\vec{\eta}_{0}-\langle\nu\rangle-\vec{\eta}_{1}-\langle\nu\rangle-\vec{\eta}_{2}-\langle\eta\rangle-\vec{\eta}_{3}$.

Proof. Apply Claim A twice to get

$$
\eta \vec{\eta}_{1} \nu \vec{\eta}_{2} \nu=\eta \vec{\eta}_{1} \eta \vec{\eta}_{2} \eta=\nu \vec{\eta}_{1} \nu \vec{\eta}_{2} \eta
$$


From Claim B it follows that $\vec{\eta}$ equals in $G$ some other word $\vec{\eta}^{\prime}$ such that if we delete in $\vec{\eta}^{\prime}$ the fragments not containing $\nu$ or $\eta$, then we obtain a word of the form

(1) $\eta^{2 \iota} \vec{\nu}$ or

(2) $\nu^{2 \iota} \vec{\nu}$, where $\vec{\nu}=\langle\nu, \eta, \nu, \eta, \ldots\rangle$ or $\vec{\nu}=\langle\eta, \nu, \eta, \nu, \ldots\rangle$ and $\iota \in \mathbf{Z}, \iota \geq 0$.

By Claim A we can replace $\eta^{2 \iota}$ by $\nu^{2 \iota}$. There are two cases.

CASE I. $\iota(\vec{\nu})$ is odd.

In this case the first and the last member of $\vec{\nu}$ are the same. Assume w.l.o.g. that $\vec{\nu}=\langle\nu\rangle \frown \vec{\nu}^{\prime} \frown\langle\nu\rangle$ and (2) holds. Let $\vec{\eta}^{\prime}=\vec{\eta}_{0} \frown\langle\nu\rangle \frown \vec{\eta}_{1} \frown\langle\nu\rangle \frown \vec{\eta}_{2}$ where $\nu, \eta \notin$ $\operatorname{Rng}\left(\vec{\eta}_{0}-\vec{\eta}_{2}\right)$. Then the inner automorphism $\varphi$ defined by

$$
\varphi(x)=\nu \vec{\eta}_{2} x\left(\nu \vec{\eta}_{2}\right)^{-1}
$$

maps $\alpha^{k} \vec{\eta}^{\prime}$ onto $\alpha^{k} \nu \vec{\eta}_{2} \vec{\eta}_{0} \nu \vec{\eta}_{1}=\alpha^{k} \vec{\eta}^{\prime \prime}$.

Now when we delete in $\vec{\eta}^{\prime \prime}$ all letters distinct from $\nu, \eta$, we obtain the word $\nu^{2 \iota+2} \vec{\nu}^{\prime}$, and $\iota\left(\vec{\nu}^{\prime}\right)=\iota(\vec{\nu})-2$. Hence, iterating this process finitely many times, we get the word $\alpha^{\iota} \vec{\eta}_{1}$ (after possible reductions according to Lemma 2) such that $\operatorname{Rng}\left(\vec{\eta}_{1}\right) \subseteq \operatorname{Rng}(\vec{\eta})$ and $\eta \notin \operatorname{Rng}\left(\vec{\eta}_{1}\right)$ or $\nu \notin \operatorname{Rng}\left(\vec{\eta}_{1}\right)$, so in this case the induction step is done.

CASE II. $\iota(\vec{\nu})$ is even. If $\vec{\nu}=\varnothing$, we finish, for then $\eta \notin \operatorname{Rng}\left(\vec{\eta}^{\prime}\right)$ or $\nu \notin$ $\operatorname{Rng}\left(\vec{\eta}^{\prime}\right)$ and $\operatorname{Rng}\left(\vec{\eta}^{\prime}\right) \subseteq \operatorname{Rng}(\vec{\eta})$. So assume that $\vec{\nu} \neq \varnothing$.

Let us consider now the free group $G_{1}$ generated by free generators $a$ and $b$. Let $H_{1}$ be the normal subgroup of $G_{1}$ generated by the set $\left\{a^{2}, b^{2}\right\}$, and let $G_{2}=$ $G_{1} / H_{1}$. The elements of $G_{2}$ can be written down in a very simple form, namely as finite sequences of the form $a b a b a b \cdots$ or bababa $\cdots$ (the length clearly may be even or odd), and any two such sequences are equal in $G_{2}$ iff they are equal.

We define some homomorphism $f: G_{0} \rightarrow G_{2}$ (it suffices to define $F(\mu)$ for every $\left.\mu \in{ }^{\omega} 2\right)$.

(i) If $h(\langle\eta, \nu\rangle)[0] \subseteq \mu$, then let $f(\mu)=a$.

(ii) If $h(\langle\eta, \nu\rangle)[1] \subseteq \mu$, then let $f(\mu)=b$.

(iii) If neither (i) nor (ii), then let $f(\mu)=e$.

Now, from the definitions of $h$ and $H_{0}$ it follows that $H_{0} \subseteq \operatorname{Ker} f$. From the choice of $\eta, \nu$ it follows that $f\left(\alpha^{k} \vec{\eta}^{\prime}\right)$ is equal to $f(\vec{\nu})$, and $f(\vec{\nu})$ equals $(a b)^{\iota}$ or $(b a)^{\iota}$ for some $\iota>0$.

But we know that, in $G,\left(\alpha^{n} \vec{\eta}^{\prime}\right)^{2}=e$, so, in $G_{2},\left[(a b)^{\iota}\right]^{2}=(a b)^{2 \iota}=e$, a contradiction, because, in $G_{2},(a b)^{2 \iota}=e$ only when $\iota=0$.

LEMMA 5. $\left|G^{2}\right| \geq \aleph_{0}$.

ProOF. This follows immediately from Theorem 1.3, but we can give also another, more direct proof. For $\eta_{0}, \eta_{1} \in{ }^{\omega} 2, \eta_{0} \neq \eta_{1}$ let $g\left(\eta_{0}, \eta_{1}\right)$ be $\eta_{0} \mid$ $\min \left\{k: \eta_{0}[k] \neq \eta_{1}[k]\right\}$. Now if $\eta_{0} \neq \eta_{1}, \nu_{0} \neq \nu_{1}, \eta_{i}, \nu_{i} \in{ }^{\omega} 2$ and $g\left(\eta_{0}, \eta_{1}\right) \neq$ $g\left(\nu_{0}, \nu_{1}\right)$, then by choosing a suitable homomorphism $f: G_{0} \rightarrow G_{2}$ with $f\left(\eta_{0} \eta_{1}\right)^{2}$, $f\left(\nu_{0} \nu_{1}\right)^{2}$ distinct (namely one of them equal to $e$ and the other distinct from $e$ in $\left.G_{2}\right)$, we may prove as in Lemma 4 that, in $G,\left(\eta_{0} \eta_{1}\right)^{2} \neq\left(\nu_{0} \nu_{1}\right)^{2}$.

Clearly the series of Lemmas 1-5 proves Theorem 2.3.

REMARK 1. A similar argument shows that $G$ is torsion-free. 
REMARK 2. In the proof of Lemma 4 we have in fact found an algorithm deciding whether $\alpha^{n} \vec{\eta}=e$ in $G$ or not. Define

$$
h_{i j}(\vec{\eta})=\sup \{n: \vec{\eta}[i] \uparrow n=\vec{\eta}[j] \uparrow n\} \quad \text { for } i, j<\iota(\vec{\eta})
$$

(with $\sup \omega=\omega$ ). We see from the proof of Lemma 4 that if we have $\vec{\nu}, \vec{\eta}$ of the same length and, for every $i, j, i^{\prime}, j^{\prime}<\iota(\vec{\eta})=\iota(\vec{\nu})$,

$$
h_{i j}(\vec{\nu})<h_{i^{\prime} j^{\prime}}(\vec{\nu}) \quad \text { iff } \quad h_{i j}(\vec{\eta})<h_{i^{\prime} j^{\prime}}(\vec{\eta})
$$

and

$$
h_{i j}(\vec{\nu})<\omega \text { iff } \quad h_{i j}(\vec{\eta})<\omega,
$$

then, in $G, \alpha^{n} \vec{\eta}=e$ iff $\alpha^{n} \vec{\nu}=e$ (it follows from the form of the algorithm mentioned above).

DEFINITION 2.4 (SEE [ 2 OR 3]). For an infinite cardinal $\lambda$ define Ded $\lambda$ as the first cardinal $\mu$ such that there is no dense linear order of cardinality $\mu$ with a dense subset of cardinality $\lambda$.

Notice that $\lambda^{+}<\operatorname{Ded} \lambda \leq\left(2^{\lambda}\right)^{+}, \operatorname{cf}(\operatorname{Ded} \lambda)>\lambda$.

COROLlaRY 2.5. Let $\aleph_{0} \leq \lambda \leq \mu<\operatorname{Ded} \lambda$. Then there exists a torsion-free group $G$ of cardinality $\mu$ such that $\left|G^{2}\right|=\lambda$.

Proof. Consider the tree ${ }^{\leq \lambda} 2$. Then we can choose a subtree of $\leq \lambda 2$ such that it has $\mu$ branches of length $\lambda$, and when we generate on the set of these branches a group $G$ (as in Definitions 2.1 and 2.2) then the set $G^{2}$ has cardinality $\lambda$.

CONJECTURE. If $G$ is infinite and has no elements of order 2 , then $|G|<$ $\operatorname{Ded}\left(\left|G^{2}\right|\right)$.

Changing somewhat Definition 2.2 one can construct analogously for any $k>2$ a torsion-free group $G$ of power continuum with $\left|G^{k}\right|=\aleph_{0}$. (In the proof of Lemma 4 one should use instead of $G_{2}$ the group $G_{k}=G_{1} / H_{k}$, where $H_{k}$ is the normal subgroup of $G_{1}$ generated by $\left\{a^{k}, b^{k}\right\}$.) The counterpart of Corollary 2.5 holds as well.

\section{REFERENCES}

1. C. C. Chang and H. J. Keisler, Model theory, 2nd ed., North-Holland, Amsterdam, 1977.

2. S. Shelah, Stability, the f.c.p., and superstability; model theoretic properties of formulas in first order theory, Ann. Math. Logic 3 (1971), 271-362.

3. __ Classification theory, North-Holland, Amsterdam, 1978.

Mathematical Institute of the Polish ACademy of Sciences, Kopernika 18, 51-617 WROCLAW, POLAND 